\title{
Interplay of information quantifiers and the modified Jaynes-Cummings model
}

Research Article

\author{
Sayed Abdel-Khalek ${ }^{1 *}$, Angelo Plastino ${ }^{2+}$, Abdel-Shafy Fahmy Obada ${ }^{3}$ \\ 1 Department of Mathematics, Faculty of Science, Sohag University, 82524 Sohag, Egypt \\ 2 IFLP-CCT-Conicet, National University La Plata, 1900 La Plata, Argentina \\ 3 Department of Mathematics, Faculty of Science,Al-Azhar University, Cairo, Egypt
}

Received 03 February 2011 ; accepted 10 June 2011

\begin{abstract}
The dynamics of the Buck and Sukumar model (B. Buck and C. V. Sukumar, Phys. Lett. A 81,132 (1981)) is investigated using different semi-classical information-theory tools. Their interplay reveals somewhat unexpected features. A new signature for the classical-quantum barrier is encountered thereby.

PACS (2008): 42.50.-p, 42.50.Ar, 42.50.Xa, 42.50.Ct

Keywords: $\quad$ Fisher information $\cdot$ Wehrl entropy $\cdot$ Cramer-Rao bound $•$ classical-quantum limit $\cdot$ Jaynes-Cummings model

(C) Versita Sp. z o.o.
\end{abstract}

\section{Introduction}

The generation of nonclassical light and its interaction with matter are receiving intense attention in quantum optics. This interest is driven by research opportunities emerging from present control-technology regarding atoms and electromagnetic fields. The concomitant, all important details of the matter-field interaction have been thoroughly scrutinized, with the Jaynes-Cummings model (JCM) playing a pivotal role [1-4]. In spite of the JCMsimplicity, it permits a variety of generalizations, applicable to distinct environments and regimes [2]. In particular, we may mention the work of Buck and Sukumar [3] that introduced the intensity dependent JCM. Because of

*E-mail: sayedquantum@yahoo.co.uk

${ }^{\dagger}$ E-mail: plastino@fisica.unlp.edu.ar the commensurability of the Rabi frequencies arising from the model's couplings, periodic revivals emerge, absent in the original JCM, with a time-dependent state-vector that is periodic itself. As a consequence, any expectation value will share such feature, which leads to an enhancement of certain effects that would otherwise be ignored by JCM-practitioners $[4,5]$. We wish here to revisit the Buck-Sukumar model using information-theory tools (ITT) so as to be in a position to, hopefully, display interesting details of the concomitant dynamics. Our quantifiers are Wehrl-entropy and Fisher's information measure (FIM). (Von Neumann's entropy is not an indicator of localization, a very important concept, explained below, for our present purposes). Additionally, the von Neumann entropy cannot be used to measure the entanglement in mixed states. Further, it depends on the eigenvalues of the field (atomic) density matrix and in many cases it is quite difficult to calculate the eigenvalues of the field density matrix.

In the present contribution our main interest lies in in- 
vestigating the temporal evolutions of the Fisher information (FI) and Wehrl's entropy, for a single-qubit system in the presence of an intensity dependent field and also to compare Fl vs. Wherl descriptions. Why does this matter? Because these two measures describe (a) interesting semiclassical physics and (b) both classical correlations and also quantum entanglement [6-8]. The Cramer-Rao bound, an interesting indicator as well that has not been much utilized in the present context, is also studied. In addition, some intriguing glimpses at the classical-quantum frontier are obtained.

The paper is organized as follows: Section 2 deals with preliminary matters: the basic model of a single-qubit in the presence of an intensity dependent field together with the Wehrl entropy fundamentals, on the one hand, and the different Fls used here on the other one. Section 3 is devoted to the discussion of our numerical results and some conclusions are drawn in Section 4. A brief historical summary of relevant previous work is presented in the Appendix.

\section{Preliminary materials}

\subsection{The model}

We review below the main details for the treatment of a two-level atom interacting with a single-mode of the cavity field [5]. The Hamiltonian, in the rotating wave approximation, can be written as [5]

$$
\begin{array}{r}
\hat{H}=\omega_{F} \hat{a}^{\dagger} \hat{a}+\frac{\omega_{A}}{2}(|0\rangle\langle 0|-| 1\rangle\langle 1|) \\
+\lambda f\left(\hat{a}^{\dagger} \hat{a}\right)\left(\hat{a}^{\dagger}|1\rangle\langle 0|+\hat{a}| 0\rangle\langle 1|\right),
\end{array}
$$

where $\omega_{F}$ is the field frequency, $\omega_{A}$ the transition frequency between the upper $|0\rangle$ and lower state $|1\rangle$ of the atom, and $\lambda$ the effective coupling constant. The field creation (annihilation) operator is $\hat{a}^{\dagger}(\hat{a})$ while $f\left(\hat{a}^{\dagger} \hat{a}\right)$ represents the intensity dependent function of the cavity field mode. Restricting ourselves to the functional form $f(\hat{n})=\sqrt{\hat{a}^{\dagger} \hat{a}}$, the interaction Hamiltonian reads

$$
\hat{H}_{l}=\lambda\left(\hat{\psi}^{\dagger}|1\rangle\langle 0|+| 0\rangle\langle 1| \hat{\psi}\right)
$$

where $\hat{\psi}=\hat{a} \sqrt{\hat{a}^{+} \hat{a}}$ and $\hat{\psi}^{\dagger}=\sqrt{\hat{a}^{+} \hat{a}} \hat{a}^{\dagger}$. The time evolution operator for the effective Hamiltonian (2) becomes

$U^{\dagger}(t)=e^{-i \hat{H}_{1} t}=\left[\begin{array}{cc}\cos \left(T \sqrt{\hat{\psi} \hat{\psi}^{\dagger}}\right) & i \frac{\sin \left(T \sqrt{\hat{\psi} \hat{\psi}^{+}}\right)}{\sqrt{\hat{\psi} \hat{\psi}^{\dagger}}} \hat{\psi} \\ i \frac{\sin \left(T \sqrt{\hat{\psi}^{+} \hat{\psi}}\right)}{\sqrt{\hat{\psi}^{+} \hat{\psi}}} \hat{\psi}^{+} & \cos \left(T \sqrt{\hat{\psi}^{+} \hat{\psi}}\right)\end{array}\right]$.

In this last relation $T=\lambda t$ is the scaled time. The time units are given by the inverse of the coupling constant $\lambda$. We assume (I) that the initial state of the system is the product $\rho^{A F}(0)=\rho^{A}(0) \otimes \rho^{F}(0)$, with our qubit assigned initially to the upper state, i.e., $\rho^{A}(0)=|0\rangle\langle 0|$, while (II) the field's initial state is a coherent-one $\rho^{F}(0)=|\alpha\rangle\langle\alpha|=$

$$
\begin{aligned}
& \sum_{n, m=0}^{\infty} C_{n}(\alpha) C_{m}^{*}(\alpha)|n\rangle\langle m| \text {, with } \\
& \qquad C_{n}(\alpha)=\alpha^{n} \sqrt{\frac{e^{-|\alpha|^{2}}}{n !} .}
\end{aligned}
$$

The Husimi $Q$-function $Q_{F}$ of the field-mode, in terms of the diagonal elements of the density operator in the coherent-state basis, is

$$
Q_{F}(\beta, t)=\frac{1}{\pi} \operatorname{Tr}_{A}\left\langle\beta\left|U(t) \rho^{A F}(0) U^{\dagger}(t)\right| \beta\right\rangle,
$$

where $\operatorname{Tr}_{A}$ means that we trace over the atomic variables. Next, we turn our attention to the semiclassical Wehrlentropy $[12,13]$ that describes the time evolution of a quantum system in phase-space. This entropy, introduced as the classical entropy of a quantum state, yields meaningful insights into the dynamics of the system $[12,13]$ and is defined as the coherent-state representation of the density matrix $[12,13,17]$ via

$$
S_{W}(t)=-\int Q_{F}(\beta, t) \ln Q_{F}(\beta, t) d^{2} \beta,
$$

where $d^{2} \beta=|\beta| d|\beta| d \Theta$. We can specialize things by recourse to the Wehrl phase distribution (Wehrl PD), defined to be the phase density of the Wehrl entropy $[18,19,22]$, i.e.,

$$
S_{\Theta}(t)=-\int Q_{F}(\beta, t) \ln Q_{F}(\beta, t)|\beta| d|\beta|
$$

where $\Theta=\arg (\beta)$. Here, the "phase space" of the problem has coordinates $(\beta, \Theta)$. The Wehrl entropy was intended by Wehrl to be primarily a measure of localization in phase space $[12,13]$. The opposite effect is called delocalization $[47,48]$. 


\subsection{Fisher Information}

The Fisher information measure (FIM) for any PDF $f(x)$ can be cast in the fashion $[31,32]$

$$
I=\int d \mathbf{x} f(\mathbf{x})\left\{\frac{\partial \ln f(\mathbf{x})}{\partial \mathbf{x}}\right\}^{2}
$$

and is encountered in many physical applications (see, for instance, [33-48], and references therein). The FIM associated to Husimi distributions $Q_{F}\left(X_{1}, X_{2}, t\right)$ is defined as [49]

$$
\begin{gathered}
I_{F}(t)=\frac{1}{2 \pi \hbar} \int_{-\infty}^{\infty} \int_{-\infty}^{\infty} Q_{F}\left(X_{1}, X_{2}, t\right) \Gamma\left(X_{1}, X_{2}, t\right) d X_{1} d X_{2} \\
=\int Q_{F}(\beta, t) \Gamma(\beta, t) d^{2} \beta,
\end{gathered}
$$

where $\Gamma\left(X_{1}, X_{2}, t\right)$ and $\Gamma(\beta, t)$ can be written in terms of the phase space parameters, yielding

$$
\Gamma\left(X_{1}, X_{2}, t\right)=\sum_{j=1}^{2}\left(\sigma_{X_{j}}(t) \frac{\partial \ln \left(Q_{F}\left(X_{1}, X_{2}, t\right)\right)}{\partial_{X_{j}}}\right)^{2}
$$

It is shown in reference [30] that in the present circumstance one has

$$
\begin{gathered}
\Gamma(\beta, t)=\sum_{j=1}^{2} \sigma_{X_{j}}^{2}(t) \\
\sum_{k=1}^{2}\left(\frac{\cos \left(\Theta+\frac{\pi}{j}-\frac{\pi}{k}\right)}{[k-2+\beta(k-1)]} \frac{\partial \ln (Q(\beta, t))}{\partial[\beta(k-2)+\Theta(k-1)]}\right)^{2},
\end{gathered}
$$

with

$$
\sigma_{X_{j}}(t)=\sqrt{\left\langle X_{j}(t)^{2}\right\rangle-\left(\left\langle X_{j}(t)\right\rangle\right)^{2}}
$$

and

$$
\left\langle X_{j}\right\rangle=\int_{-\infty}^{\infty} \int_{-\infty}^{\infty} X_{j} Q\left(X_{1}, X_{2}, t\right) d X_{1} d X_{2}
$$

We also consider, as a dynamical measure, the quantity

$$
l_{\Theta}(t)=-\int_{0}^{\infty} Q_{H}(\beta, t) \Gamma(\beta, t)|\beta| d|\beta|
$$

It is worth noting that the definition (8) is given in analogy to that of the field Wehrl entropy (the special case $t=0$ being $I_{F}(0)=2$ ) so that the corresponding Fisher's phase distribution can be cast, in terms of the error function $\operatorname{erf}(x)=\frac{2}{\sqrt{\pi}} \int_{0}^{x} e^{-y^{2}} d y$, as

$$
\begin{array}{r}
l_{\Theta}(0)=\frac{1}{2 \pi} \exp \left(x^{2}-\alpha^{2}\right) \times \\
\left\{x \sqrt{\pi}[1+\operatorname{erf}(\mathrm{x})] f_{1}+\exp \left(-x^{2}\right) f_{2}\right\},
\end{array}
$$

where $x=\alpha \cos (\Theta)$ and

$$
f_{j}=\alpha^{2}-x^{2}+\frac{j}{2}, j=1,2
$$

In correlations terms the bipartite system becomes uncorrelated whenever $I_{F}(0) \simeq 2$, this value representing the lower bound for $I_{F}$. One has

$$
I_{F}(0)=S_{W}(0)+1-\ln \pi
$$

and

$$
\begin{aligned}
I_{\Theta}(0)= & S_{\Theta}(0)-\frac{\ln \pi}{2 \pi} \exp \left(-\alpha^{2}\right) \\
& \left\{1+x \sqrt{\pi}(1+\operatorname{erf}(x)) \exp \left(x^{2}\right)\right\} .
\end{aligned}
$$

Equations (15) - (16) establish the connection between Fisher's information measure (FIM) and Wehrl's entropy at $T=0$. Notice that at this time $I_{F}-I_{\Theta}=1-\ln \pi !=$ constant, which is a counterintuitive result, since one expects their sum to be approximately constant [48]. This curious result is due to the periodicity of the evolution.

\subsection{Cramer-Rao bound}

The "true" informational content of $\mathrm{Fl}$ is conveyed by the Cramer-Rao inequality (CR). Indeed, this is its most important property, that we recapitulate in one-dimension, for simplicity's sake. If the classical Fisher information associated with translations of a one-dimensional observable $x$ with corresponding probability density $f(x)$ is $[31,52]$

$$
I_{x}=\int \mathrm{d} x f(x)\left(\frac{\partial \ln f(x)}{\partial x}\right)^{2}
$$

then it obeys the above referred inequality, namely

$$
(\Delta x)^{2} \geq I_{x}^{-1}
$$

involving the variance of the stochastic variable $x$ [52] 


$$
(\Delta x)^{2}=\left\langle x^{2}\right\rangle-\langle x\rangle^{2}=\int \mathrm{d} x f(x) x^{2}-\left(\int \mathrm{d} x f(x) x\right)^{2} .
$$

We remark that the derivative operator significantly influences the contribution of minute local $f$-variations to $\mathrm{Fl}^{\prime}$ s value, so that the quantifier is called a "local" one. Note that Wehrl's entropy decreases with skewed distributions, while Fisher's information increases in such a case. Local sensitivity is useful in scenarios whose description necessitates appeal to a notion of "order".

For our present purposes we deal with a time-dependent $\mathrm{CR}$, that, in self-explanatory notation reads

$$
I_{F}(t) \Delta_{t}^{2}
$$

where

$$
\begin{gathered}
\Delta_{t}^{2}=\left\langle\beta^{2}\right\rangle-\langle\beta\rangle^{2} \\
\left\langle\beta^{s}\right\rangle=\int_{0}^{2 \pi} \int_{0}^{\infty}|\beta|^{s} Q_{F}(\beta, t)|\beta| d|\beta| d \Theta, s=1,2 .
\end{gathered}
$$

\section{Results}

We start now the presentation of our numerical results. We will see that the coherent state parameter $\alpha$, representing the square root of the mean-photon number, greatly influences the dynamics, as can be clearly appreciated in Figs. 1 that depict, respectively, $I_{F}$ and $S_{W}$ as a function of $T$ and $\alpha[(a)$ and (b)] together with their projections on the $\alpha-T$-plane [(c) and (d)] ( $T$ is a "scaled" time). Both the inherent periodicity of the dynamics and
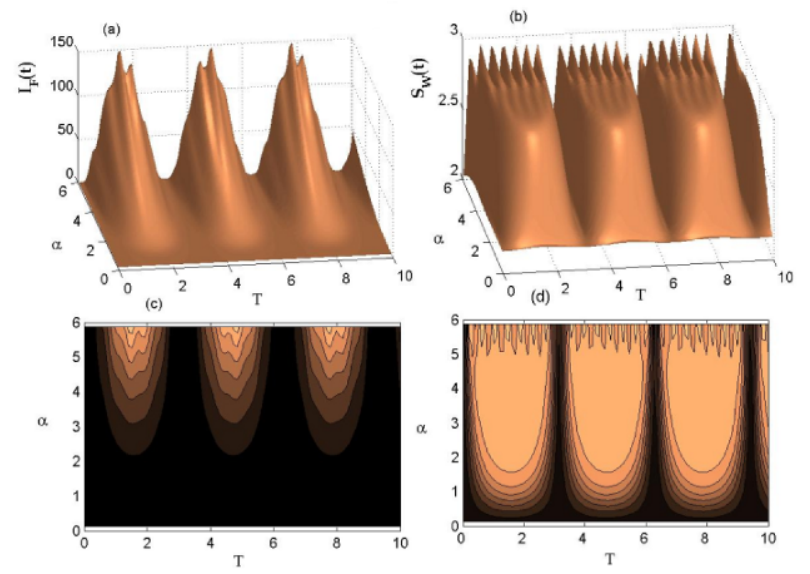

Figure 1. The time-evolution of $(\mathrm{a}, \mathrm{c})$ Fisher' information $I_{F}(t),(\mathrm{b}, \mathrm{d})$ Wehrl's entropy $S_{W}(t)$, versus the scaled time $T=\lambda t$ and the root of the mean photon number $\alpha=\sqrt{\bar{n}}$. the long living correlation between the single qubit and the coherent field are clearly visible. They increase as the photon-number grows. Both quantifiers exhibit the periodicity of the system.

Fig. 2 is the analog of 1 , but this time for the phase distributions [Cf. Eq. (12)]. It is shown that, as the time evolution proceeds, the single peak of the initial coherent state splits up into two peaks diverging away from each other gradually at the time $T=\pi$. The two peaks merge into a single peak at $\Theta= \pm \pi$. Also, when $T=2 \pi$ the two peaks become joined at $\Theta=0$. Time periodicity is evident. A comparison between Fig. 2(a, c) and Fig. 2(b,d) exhibits the same behavior, although with smaller changes ensuing at $T=m \pi$.
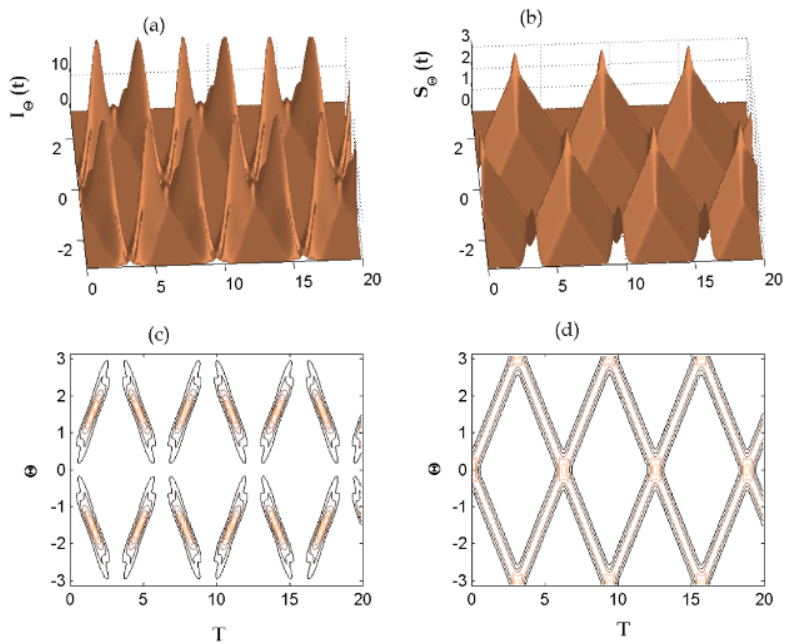

Figure 2. The Fisher's Phase Distribution $l_{\Theta}(t)$ and Wehrl's Phase Distribution $S_{\Theta}(t)$ versus the scaled time $T$ and the phase space parameter $\Theta$ for $\alpha=3$.

In figure 3 we plot the $\mathrm{Fl}$ - and Wehrl- time evolutions together with the associated $X_{2}$-variance (for typographical simplicity, we set in the graphs $Y \equiv X_{2}$ ). One chooses three values of the $\alpha$-parameter, namely, $=1,2,3$, respectively. In order to ensure good accuracy, the behavior of the Fisher information $I_{F}(t)$ has been determined using an appropriate scale so as to meaningfully compare it to Wehrl's entropy. Fl's behavior is clearly dominated by the variance component $\sigma_{X_{2}}^{2}(t)$ [Cf. Eq. (11)]. This is to be expected, and FIM is indeed a measure of fluctuations [31]. Note the quite different numerical values taken by $\mathrm{FI}$, much larger than those of $S_{W}$. Thus, the Fisherpeaks become steeper than the Wehrl-ones. The highest values attained at their peaks by both quantifiers grow with $\alpha$. Note that fluctuations also grow with $\alpha$.

Fig. 4 illustrates our two quantifiers, i.e., the $I_{F}$ vs. $S_{W}$ behavior, a plot that has to be looked at while keeping in 


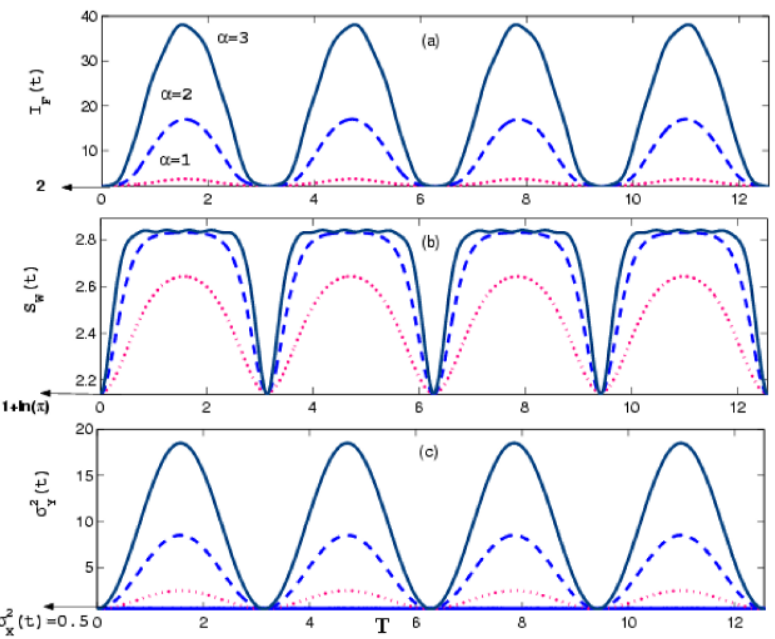

Figure 3. Time volution of (a) Fisher' information $I_{F}(t)$, (b) Wehrl's entropy $S_{W}(t)$, and (c) variances $\sigma_{X_{2}}^{2}(t)$. We set $Y \equiv X_{2}$. We also plot curves for different values of the square root of the mean photon number $\alpha$, namely, dotted curve for $\alpha=1$, dashed curve for $\alpha=2$, and solid curve for $\alpha=3$.
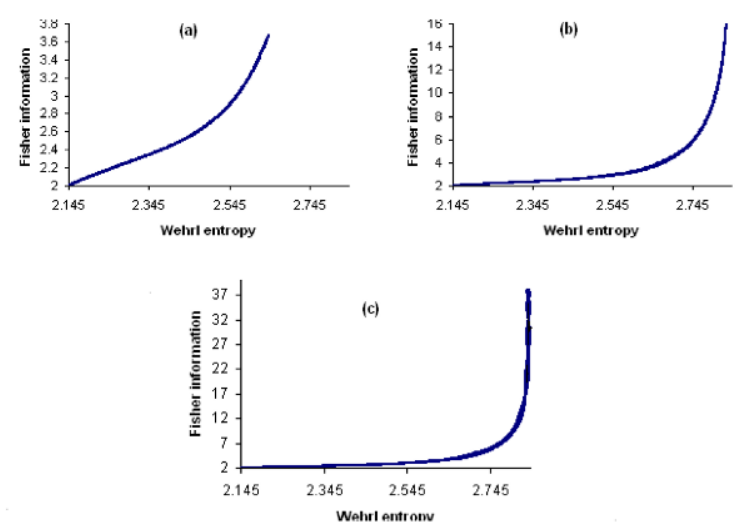

Figure 4. Illustration of the $I_{F}$ vs. $S_{W}$ behavior for parameters values for different values of the square root of $\alpha$, where figure (a) $\alpha=1$, figure (b) $\alpha=2$, and $\alpha=3$ for figure (c).

mind that of the preceding figure. Since $S_{W}$ is a global measure, while FIM is a local one [47], at first sight the behavior depicted might perhaps appear surprising, although this is not really so, on the basis of the preceding considerations related to Fig. 3. The peak-altitude grows with $\alpha$ for both quantifiers, here depicted at a fixed time. The monotonous behavior that is apparent can thus be readily understood. Remember now the delocalization effect we have mentioned above [47], associated to Wehrl's entropy. Delocalization in our $(\beta, \Theta)$ increases a bit from $\alpha=1$ to $\alpha=2$, where it stops growing. This already is an indication of classicality, that we expect for very large $\alpha$, since one way of reaching the classical limit is by going over to the thermodynamic limit of infinite particle-number. We are here encountering a seemingly bizarre scenario in which such limit is reached with just 9 particles, though. The route to classicality $[50,51]$ is thus paved by i) a growing mean photon-number (the celebrated $N \rightarrow \infty$ way of achieving classicality) and ii) a stabilization of Wehrl's entropy. Why? because delocalization stops augmenting, indeed, wanes as we approach the classical limit. Such kind of scenario begins to insinuate itself at $\alpha=2$ (four photons) and becomes fully installed already at $\alpha=3$ (nine photons)! Thus, $S_{W}$ cannot continue growing as $\alpha$ grows, but nothing impedes $I_{F}$ to increase, as the more information becomes available if the structure of the system acquires additional details because more particles are involved.
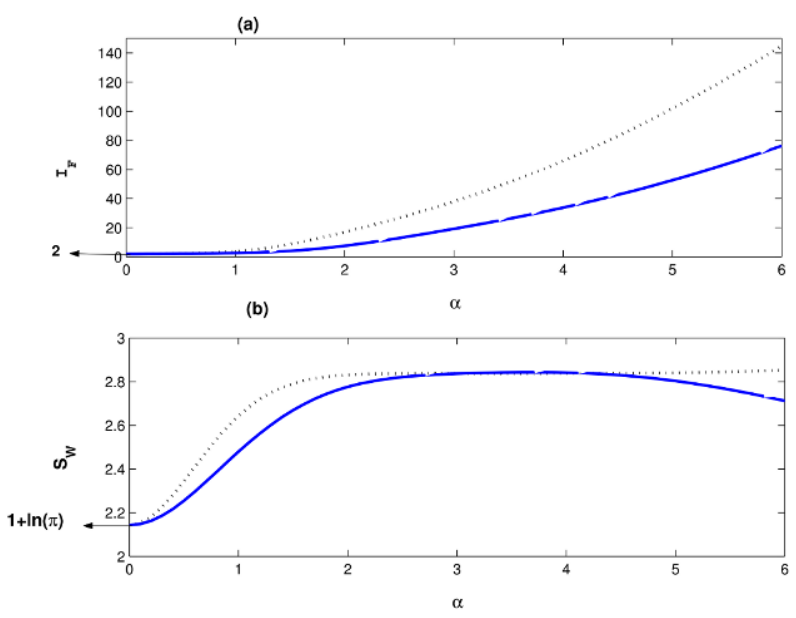

Figure 5. We depict the (a) FIM and (b) Wehrl entropy behaviors versus the square root of the mean photon number for two different values of the scaled time $T$ where $T=\pi / 4$ (solid line) and $T=\pi / 2$ (dotted line).

The above considerations receive a boost via Fig. 5, that depicts the FI-Wehrl behavior versus the mean photon number. $I_{F}$ always grows with $\alpha$ entailing that, as one intuitively understands, errors diminish as particle-number grows. Wherl's measure has a peak at about $\alpha=3$ and then diminishes. This can also be understood on the basis of preceding considerations. $S_{W}$ measures our ignorance about localization in phase-space, which, as it should, becomes smaller as $\alpha$ grows.

In Fig. 6 we display some results about the evolution of the CR given by Eq. (20). We see that the CR product oscillates with time and rapidly increases as $\alpha$ grows. At $\alpha=1$ this product almost saturates its lower bound (unity). This fact can be regarded as confirming the prevalent idea that quantum states "carry" more information than clas- 


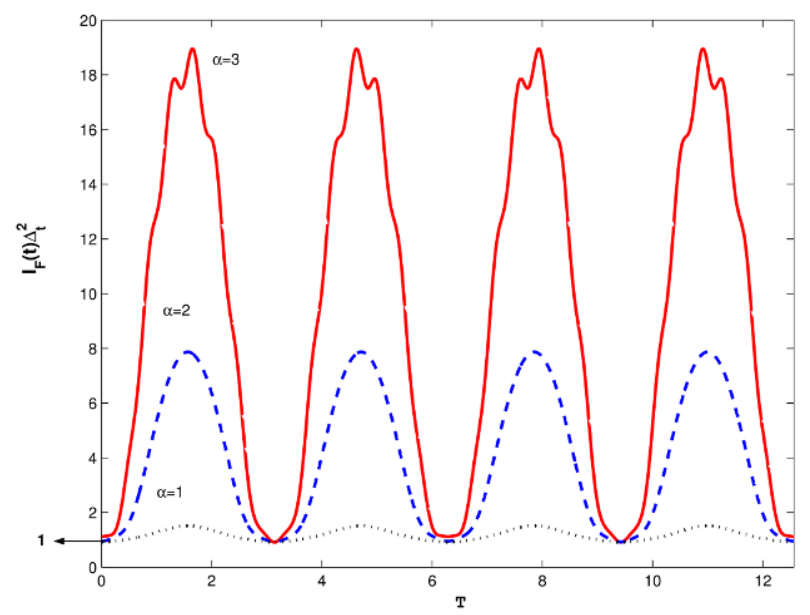

Figure 6. The Cramer-Rao product $I_{F}(t) \Delta_{t}^{2}$ as a function of the scaled time $T$ for different values of $\alpha$ where the dotted curve $\alpha=1$, dashed curve $\alpha=2$ and the solid curve $\alpha=3$.

sical ones. Why? Because the former contain, in addition to classical correlations, also the quantum ones represented by entanglement and quantum discord (see for instance [53]).

\section{Conclusions}

We have here considered, from an information-theory viewpoint, the dynamics of a single-qubit system. Our information-quantifiers were the Wehrl entropy, a phasespace localization measure and Fisher's information. These two quantities aptly illustrate on the complicated dynamics at hand. The main characteristics of the problem are governed by the mean photon number and the intensity dependent field.

An extensive numerical analysis was performed and illustrated via a variety of graphs. As one could foretell, periodicity is a main feature. Long-lived correlations between the qubit system and the coherent field are clearly appreciated. The monotonous (with $\alpha$ ) growth of the Fisher measure as the Wehrl entropy grows is a counterintuitive feature that has been detected. This is a surprising facet because it is well known that whenever Fisher information grows, Shannon or Wehrl entropies decrease [31, 48]. Notice that FI measures gradient content [31, 48] while Wehrl's measure is a localization-indicator [48]. However, the physics of the phenomenon can be understood. As explained in the preceding Section, the interplay between our two quantifiers $S_{W}$ and $I_{F}$ is equivalent to that of localization (Wehrl) vs. diminution of errors (Fisher). Rather unexpectedly, we get illuminating insights into the emergence of classicality, in line with very recent findings [54]. We seem to have discovered a new signature of the classical-quantum barrier: a rapid growth of $I_{F}$ at constant $S_{W}$.

\section{Appendix: Background material}

\section{Generalities}

This is an historical Section that can be omitted at a first reading. Information-theory tools ITT have also been the subject of much interest, in particular when they are applied in a non-thermal setting. In this regard, von Neumann's (NE) [9], linear (LE) [10], and Shannon's entropy (SE) [11] have been frequently used for a variety of quantum systems. It is worth mentioning that the SE involves only the diagonal elements of the pertinent density matrix and in some cases yields information similar to that obtained from either the NE or LE measures. Other important entropic-scenario involve semiclassical physics and one employs there the phase-space field Wehrl entropy (FWE) $[12,13]$.

\section{Some relevant previous work on quantum op- tics}

The FWE has been successfully applied in descriptions of different properties of quantum optical fields, such as phase-space uncertainty $[14,15]$, decoherence $[16,17]$, etc., a theme that will be the focus of our endeavor in this work. As a consequence, we are led to the concept of Wehrl phase distribution (WPD), that has been extensively developed and shown to be a successful indicator of both noise (phase-space uncertainty) and phase randomization $[18,19]$. Furthermore, the FWE has been fruitfully applied to dynamical systems. In this respect we must mention that the FWE-time evolution in the case of the Jaynes-Cummings model has been thoroughly investigated in $[17,20,21]$. The FWE i) turns out to be more apt for distinguishing amongst states than the NE $[18,19]$ and ii) is known to yield helpful information on atomic inversion processes. Indeed, FWE-studies of the canonical setting in which a single-trapped ion interacts with a laser field (with different field configurations) have been considered in [22]. We also know now that both (1) the fluctuations of the laser phase and (2) the initial-state setting play important roles concerning the evolution of quantifiers like the Husimi $Q$-function, Wehrl's entropy and Wehrl's phase distribution [22].

A rather different functional of the probability distribution function (PDF), called Fisher's information (FI) [23] 
was also invoked here. FI was originally introduced by Fisher [23] as a measure of "intrinsic accuracy" in statistical estimation theory. We will concern ourselves in this communication with the Fl-version constructed with the semi-classical Husimi probability distribution function (PDF) [24-26]. It has been shown in [27] that FI can be used for evaluating the accuracy limits of a quantum measurement because it provides one with meaningful error estimates, even in the case of highly nonclassical regimes. This is due to the fact that variances are used to quantify the error in quantum measurements (variances and $\mathrm{FI}$ are intimately linked via the Cramer-Rao bound [23]). The relation between the so-called atomic Fisher information (AFI) and different entanglement measures such as von Neumann's, linear, and atomic Wehrl's entropy has been analyzed in [28], whose authors found that the entanglement of a two-level atom can be measured by them. Also, $\mathrm{FI}$ is used to measure the correlation between the quantized field and a Kerr medium [29]. A still new application for $\mathrm{Fl}$ is found in [30]: it can be employed as an information quantifier for the description of the weak field versus strong field dynamics in the case of a trapped ion in a laser field. Ref. [30] compared FI, as an information quantifier, with von Neumann's and Wehrl's entropies, and provided some analytical $\mathrm{FI}$-results.

\section{References}

[1] E. T. Jaynes, F. W. Cummings, IEEE 51, 89 (1963)

[2] B. W. Shore, P. L. Knight, J. Mod. Opt. 40, 1195 (1993)

[3] B. Buck, C. V. Sukumar, Phys. Lett. A 81, 132 (1981)

[4] K. Zaheer, M. R. B. Wahiddin, J. Mod. Opt. 41, 150 (1994)

[5] D. S. Freitas, A. Vidiella-Barranco, J. A. Roversi, Phys. Lett. A 249, 275 (1998)

[6] J. Batle, A. R. Plastino, M. Casas, A. Plastino, Phys. Lett. A 318, 506 (2003)

[7] J. Batle, M. Casas, A. R. Plastino, A. Plastino, Int. J. Quantum Inf. 3, 99 (2005)

[8] J. Batle, M. Casas, A. Plastino, A. R. Plastino, Phys. Rev. A 71, 024301 (2005)

[9] J. von Neumann, Mathematical Foundations of Quantum Mechanics (Princeton University Press, Princeton, 1955)

[10] G. Manfredi, M. R. Feix, Phys. Rev. E 62, 4665 (2000)

[11] C. E. Shannon, W. Weaver, The Mathematical Theory of Communication (Urbana University Press, Chicago, 1949)

[12] A. Wehrl, Rev. Mod. Phys. 50, 221 (1978)

[13] A. Wehrl, Rep. Math. Phys. 30, 119 (1991)

[14] V. Bužek, C. H. Keitel, P. L. Knight, Phys. Rev. A 51,
2575 (1995)

[15] J. B. Watson et al., Phys. Rev. A 54, 729 (1996)

[16] A. Anderson, J. J. Halliwell, Phys. Rev. D 48, 2753 (1993)

[17] A. Orlowski, H. Paul, G. Kastelewicz, Phys. Rev. A 52, 1621 (1995)

[18] A. Miranowicz, H. Matsueda, M. R. B. Wahiddin, J. Phys. A: Math. Gen. 33, 5159 (2000)

[19] A. Miranowicz et al., J. Phys. A: Math. Gen. 34, 3887 (2001)

[20] A-S F. Obada, S. Abdel-Khalek, J. Phys. A: Math. Gen. 37, 6573 (2004)

[21] S. Abdel-Khalek, Physica A 387, 779 (2008)

[22] S. Abdel-Khalek, Phys. Scr. 80, 045302 (2009)

[23] R. A. Fisher, Proc. Cambridge Philos. Soc. 22, 700 (1925)

[24] F. Pennini, A. Plastino, Phys. Rev. E 69, 057101 (2004)

[25] F. Pennini, A. Plastino, Phys. Lett. A 326, 20 (2004)

[26] F. Pennini, A. Plastino, G. L. Ferri, F. Olivares, Phys. Lett. A 372, 4870 (2008)

[27] Z. Hradil, J. J. Rehacek, Phys. Lett. A 334, 267 (2005)

[28] A.-S. F. Obada, S. Abdel-Khalek, Physica A 389, 891 (2010)

[29] S. Abdel-Khalek, Int. J. Quantum Inf. 7, 1541 (2009)

[30] A.-S. F. Obada, S. Abdel-Khalek, A. Plastino, Physica A 390, 525 (2011).

[31] B. R. Frieden, Physics from Fisher information (Cambridge University Press, Cambridge, 1998); Science from Fisher information (Cambridge University Press, Cambridge, 2004)

[32] D. A. Lavis, R. F. Streater, Studies in the History and Philosophy of Modern Physics 33, 327 (2002)

[33] V. Kapsa, L. Skala, J. Chen, Physica E 42, 293 (2010)

[34] B. R. Frieden, B. H. Soffer, Physica A 388, 1315 (2009)

[35] M. R. Ubriaco, Phys. Lett. A 373, 4017 (2009)

[36] S. Lopez-Rosa, J. C. Angulo, J. S. Dehesa, R. J. Yanez, Physica A 387, 2243 (1998)

[37] S. P. Flego, F. Olivares, A. Plastino, M. Casas, Entropy 13, 184 (2011)

[38] K. D. Sen, J. Antolin, J. C. Angulo, Phys. Rev. A 76, 032502 (2007)

[39] A. Nagy, Chem. Phys. Lett. 449, 212 (2007)

[40] A. Nagy, Chem. Phys. Lett. 425, 154 (2006)

[41] S. Curilef, F. Pennini, A. Plastino, G. L. Ferri, J. Phys. A 134, 012029 (2008)

[42] A. Hernando, C. Vesperinas, A. Plastino, Physica A 389, 490 (2010)

[43] A. Hernando, C. Vesperinas, A. Plastino, Phys. Lett. A 374, 18 (2009)

[44] F. Pennini, G. L. Ferri, A. Plastino, Entropy 11, 972 (2009) 
[45] F. Olivares, F. Pennini, A. Plastino, Physica A 389, 2218 (2010)

[46] F. Pennini, A. Plastino, B. H. Soffer, C. Vignat, Phys. Lett. A 373, 817 (2009)

[47] F. Pennini, A. Plastino, Phys. Lett. A 365, 263 (2007)

[48] F. Pennini, A. Plastino, Phys. Rev. E 69, 057101 (2004)

[49] F. Pennini, A. Plastino, Phys. Lett. A 326, 20 (2004).

[50] A. Kowalski, M. T. Martin, L. Zunino, A. Plastino, Phys. Lett. A 374, 1819 (2010)
[51] A. Kowalski, A. Plastino, Physica A 388, 4061 (2009)

[52] M. J. W. Hall, Phys. Rev. A 62, 012107 (2000); A. L. Mayer, C. W. Pawlowski, H. Cabezas, Ecological Modeling 195, 72 (2006)

[53] B. Batle, A. Plastino, A. R. Plastino, M. Casas, arXiv:quant-ph/1103.0704

[54] Xia-Ji Liu, Hui Hu, P. D. Drummond, Phys. Rev. A 82, 023619 (2010) 